\title{
A Busbar Current Sensor With Frequency Compensation
}

\author{
Pavel Ripka, Václav Grim, and Vojtěch Petrucha \\ Faculty of Electrical Engineering, Czech Technical University in Prague, 16627 Praha, Czech Republic
}

DC/AC yokeless galvanically insulated electric current sensors are required for applications, e.g., in automotive and aerospace engineering, where size, weight, and/or price are strictly limited. A busbar current sensor with differential fluxgate in the hole has $1000 \mathrm{~A}$ range and $10 \mathrm{~mA}$ resolution. Using an asymmetric shape, we achieved a frequency error below $\pm 3 \% \mathrm{up}$ to $1 \mathrm{kHz}$, while keeping high temperature stability and low sensitivity to mechanical misalignments. The $2.5 \mathrm{~mA} /{ }^{\circ} \mathrm{C} \mathrm{maximum} \mathrm{dc} \mathrm{drift} \mathrm{is} \mathrm{four}$ times better than when using an AMR sensor and 1000 times better than when using a Hall sensor. The sensor linearity error is below $0.1 \%$.

Index Terms-Current sensor, fluxgate, magnetic sensor.

\section{INTRODUCTION}

C OMPACT yokeless current sensors are small, lightweight, and cheap. They are used in mobile and embedded applications, and for measuring high dc/ac currents, for which a magnetic core would be too large [1]-[3].

\section{A. Busbar Sensor With Magnetic Sensors on the Surface}

Conventional busbar sensors use a pair of Hall sensors on the conductor surface [4], [5]. Differential configuration partly suppresses the external fields. A current range of $10 \mathrm{kA}$ is easily achievable [6], but the sensor has high offset drift. A current sensor based on magnetostriction has a similar problem with stability [7]. The use of an integrated fluxgate allows us to increase the range of the sensor to $600 \mathrm{~A}$ with a similar offset stability and noise. A disadvantage of current sensors of this type is their high sensitivity to the distance between the sensor and the conductor surface, which changes due to temperature dilatation. Our experiments have shown that a $0.1 \mathrm{~mm}$ shift of the sensor causes a $2 \%$ change in sensitivity. Another disadvantage of this type of current sensor is its very high frequency dependence: for a magnetic sensor directly on the surface of the busbar, the sensitivity at $1 \mathrm{kHz}$ drops to $12 \%$ of the dc sensitivity.

\section{B. Busbar Sensor With Magnetic Sensors in the Hole}

A dc/ac current sensor with a differential integrated fluxgate inside the busbar is described in [8]. An advantage of this solution is that the range can easily be adjusted by changing the distance of the sensor from the busbar center, where the sensitivity is zero.

A similar busbar sensor with a range of $300 \mathrm{~A}$ is described in [9]. It uses an AMR sensor bridge in a semi-cylindrical slot in the busbar. Unlike the sensor described in [9], we use a differential sensor, which suppresses the influence of external currents and magnetic fields much more effectively.

Manuscript received August 9, 2016; revised October 18, 2016; accepted October 20, 2016. Date of publication October 25, 2016; date of current version March 16, 2017. Corresponding author: P. Ripka (e-mail: ripka@fel.cvut.cz).

Color versions of one or more of the figures in this paper are available online at http://ieeexplore.iee.org.

Digital Object Identifier 10.1109/TMAG.2016.2620959
The busbar sensor with a hole has the advantage over a sensor on the surface that the frequency dependence is lower. With a cylindrical hole, the frequency error is $14 \%$, while for amphitheater geometry, the error was reduced to 9\% [10]. Problems with amphitheater geometry were the large sensitivity to a geometrical mismatch, and increased manufacturing complexity.

This paper presents the new shape of the busbar and the optimization of the sensor position, which led to $\pm 3 \%$ frequency error from dc to $1 \mathrm{kHz}$. Sensitivity to temperature dilatation and geometrical mismatch is also analyzed.

All electromagnetic field simulations were performed in Ansys Maxwell using a 3-D eddy current solver and adaptive meshing. The final solution uses approximately $600 \mathrm{k}$ tetrahedra. Effects of heating were examined by co-simulation between Maxwell 3-D (to calculate losses) and Ansys Mechanical (to get temperature distribution).

\section{A. Differential Fluxgate Sensor}

For the current sensor, we use the integrated fluxgate DRV425, manufactured by Texas Instruments [11]. The main advantage of this sensor is its low offset drift with temperature $5 \mathrm{nT} /{ }^{\circ} \mathrm{C}$ compared with AMR $\left(20 \mathrm{nT} /{ }^{\circ} \mathrm{C}\right)$ and the Hall sensor $\left(5 \mu \mathrm{T} /{ }^{\circ} \mathrm{C}\right)$.

Two fluxgate sensors were connected in a differential mode. Each sensor is individually feedback compensated, and we process the difference between the compensation currents. All the necessary electronics is integrated inside the sensor chips. The only external components are the sensing resistors. The compensation current flowing through the microfabricated solenoid compensation coil is in the range of $10 \mathrm{~mA}$ for the measured current of $1000 \mathrm{~A}$. This high ratio cannot be achieved by a fluxgate-based ac/dc current transformer, due to the high parasitic capacitance of the secondary winding [12].

The two fluxgate sensors are mounted on the opposite sides of the printed circuit board. The effective distance between the sensors was $2.7 \mathrm{~mm}$.

\section{B. Busbar Geometry}

Fig. 1 shows the electric current distribution inside the $60 \mathrm{~mm} \times 10 \mathrm{~mm}$ conductor and the magnetic field in the free air for a central cylindrical hole $19 \mathrm{~mm}$ in diameter. While 

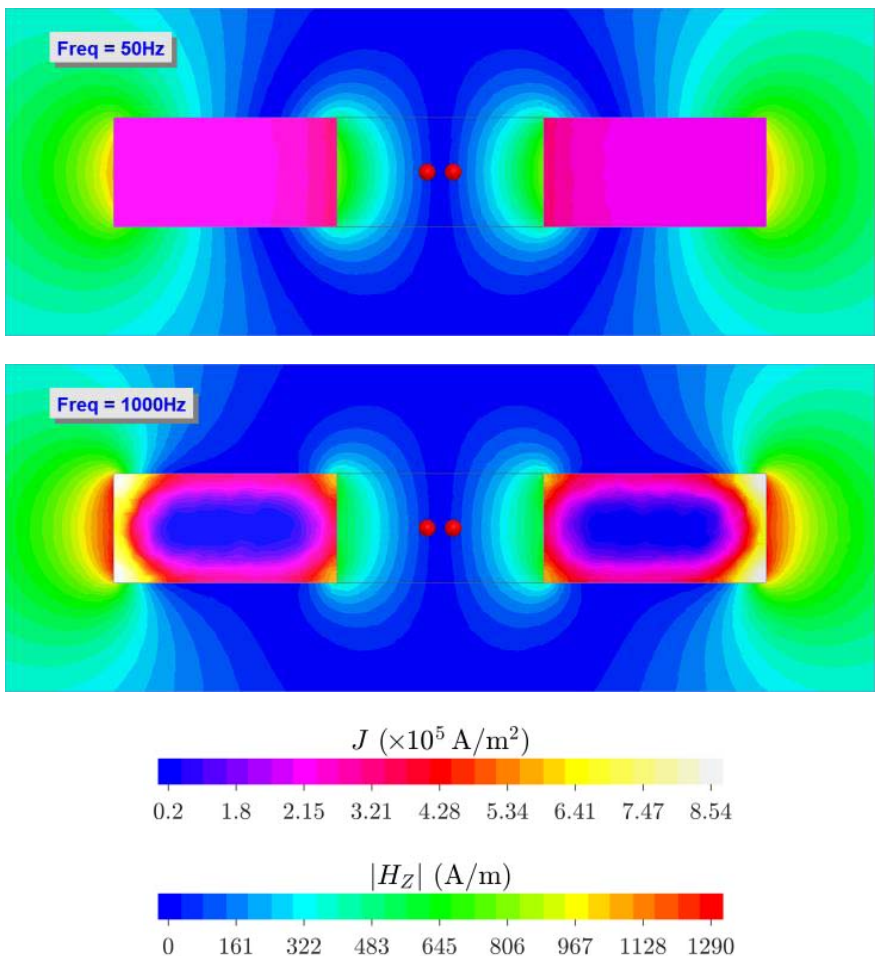

Fig. 1. Electric current distribution inside the $60 \mathrm{~mm} \times 10 \mathrm{~mm}$ conductor and the magnetic field in the free air for a central cylindrical hole $19 \mathrm{~mm}$ in diameter. The FEM simulation was performed for $f=50 \mathrm{~Hz}$ and $1 \mathrm{kHz}$. Red dots: sensor positions.

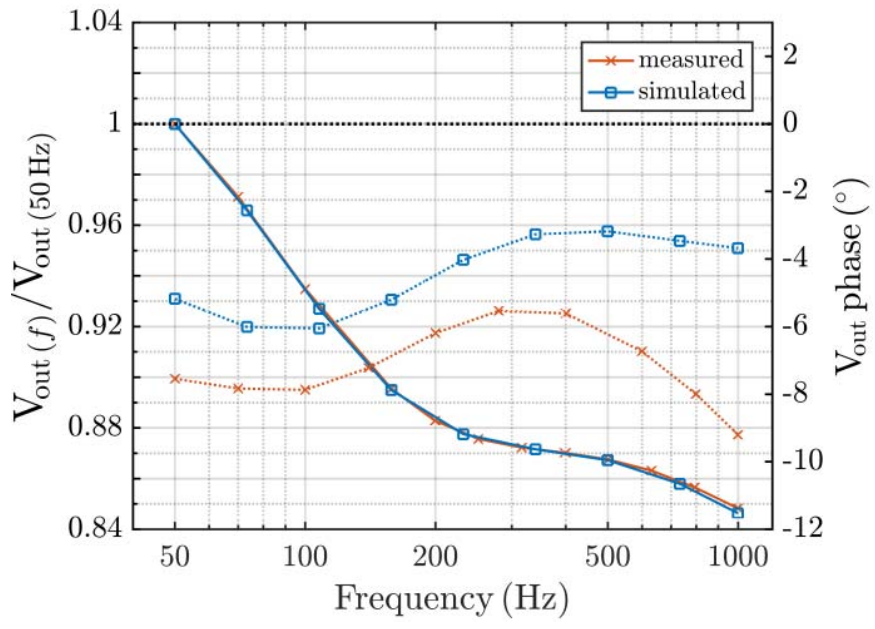

Fig. 2. Frequency dependence of the busbar current sensor with the traditional symmetrical design (measurement and 3-D simulation). Full line: amplitude characteristics. Dotted line: phase characteristics.

the current is very homogeneous for a frequency of $50 \mathrm{~Hz}$, at $1 \mathrm{kHz}$, the effect of an eddy current increased the current density at the external corners by a factor of 1.5 . As these regions are further away from the sensors, this results in a decreased sensitivity of the sensor.

The frequency dependence as a result of 3-D simulation and measurement is shown in Fig. 2 for a differential magnetic sensor having a gradiometric distance of $2.7 \mathrm{~mm}$. The frequency error of $16 \%$ at $1 \mathrm{kHz}$ should be compared with the $32 \%$ error of the transducer based on Hall sensors on the surface of the busbar [5]. The phase error at $1 \mathrm{kHz}$ is $10^{\circ}$,

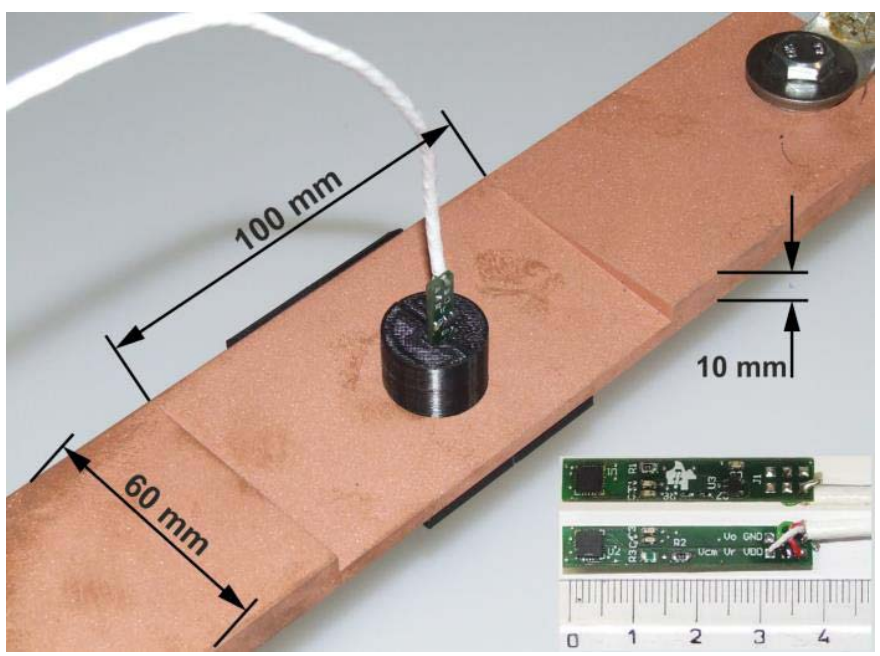

Fig. 3. New busbar sensor with a wedge-shaped profile. The circular hole is located asymmetrically and the position of the sensors in the hole is also asymmetrical.

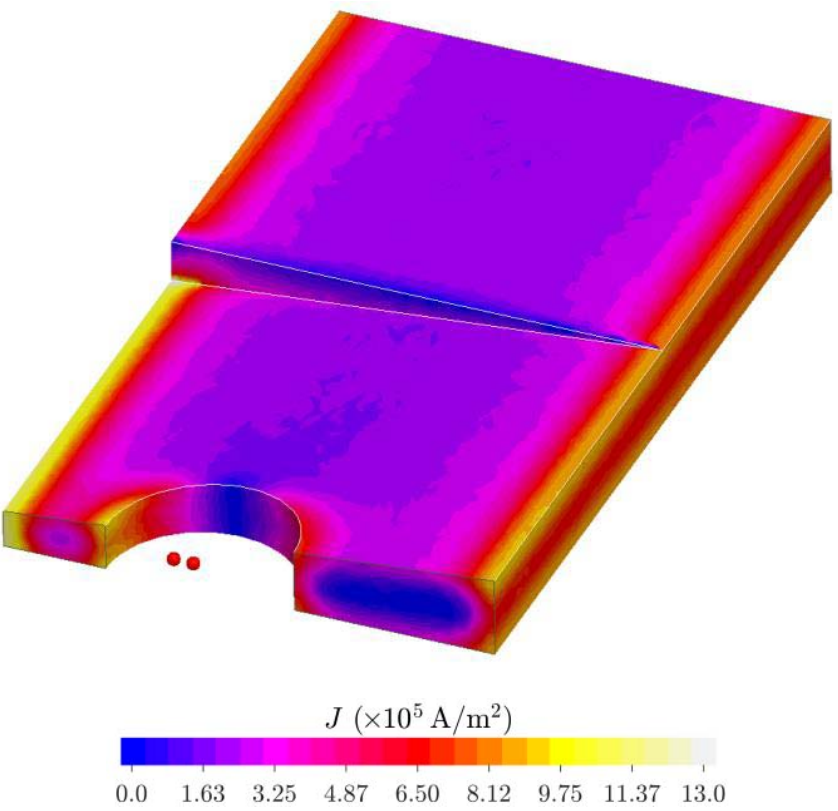

Fig. 4. Electric current distribution in the new busbar sensor at $1 \mathrm{kHz}$ Red dots: sensor positions.

which is too large for this sensor to be used for power and energy measurements. We attribute the difference between the simulated and measured phase characteristics to error in simulation, as we observed negligible phase error of the sensor itself at low frequencies.

In order to better compensate the frequency dependence, we analyzed a range of alternative geometries. Based on 3-D Finite-Element Modelling (FEM) simulations, we selected an asymmetric design with a wedge bar. The new sensor is shown in Fig. 3. The dimensions were selected, so that the sensitivity is approximately $1 \mathrm{mV} / \mathrm{A}$. As the fluxgate sensitivity is $488 \mathrm{mV} / \mathrm{mT}(12.2 \mathrm{~mA} / \mathrm{mT}$ with a $10 \Omega$ sensing resistor and an instrumentation amplifier with gain of 4), the corresponding field factor is $500 \mathrm{~A} / \mathrm{mT}$.

Fig. 4 shows the 3-D FEM simulation of the current distribution of the new asymmetric design. Because of the 

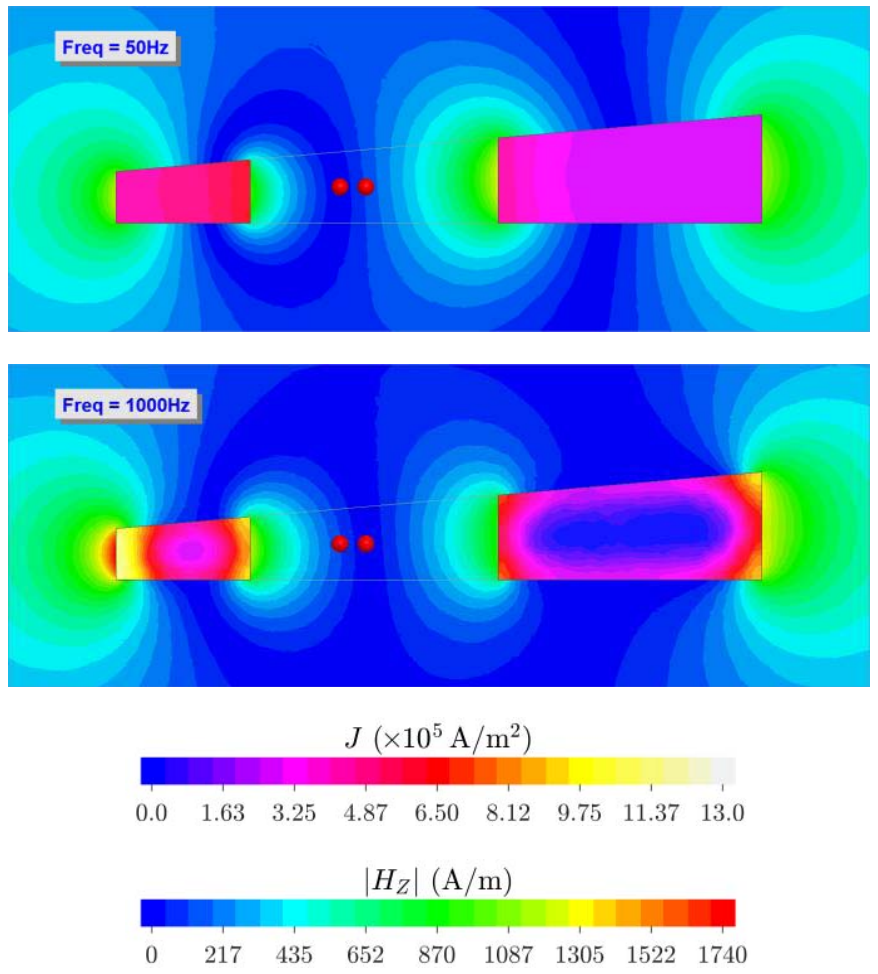

Fig. 5. Current and field distribution in the new busbar sensor with a wedgeshaped profile. 3-D FEM simulation at $50 \mathrm{~Hz}$ and $1 \mathrm{kHz}$. Red dots: sensor position.

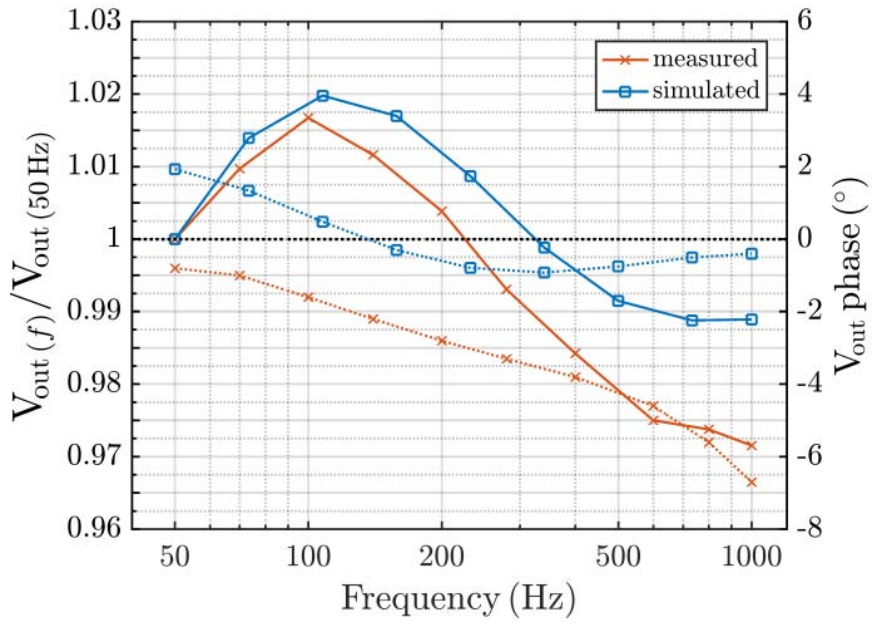

Fig. 6. Frequency dependence of the busbar current sensor with the new asymmetrical design (measurement and 3-D simulation). Full line: amplitude characteristics. Dotted line: phase characteristics.

modified shape, the current is even more redistributed due to the eddy currents. Fig. 5 shows the current and magnetic field distribution in the central plane, where both magnetic sensors are located. It is clear that the field gradient is more frequency dependent than the previous geometry. We solved the task of selecting the position of the differential sensor pair to minimize the frequency dependence while keeping a reasonable conversion factor and low sensitivity to misalignment. This optimization was made by parametric FEM simulation. The selected sensor locations are marked by red dots.

The measured frequency characteristics shown in Fig. 6 confirmed the expectations from the simulations. The measured

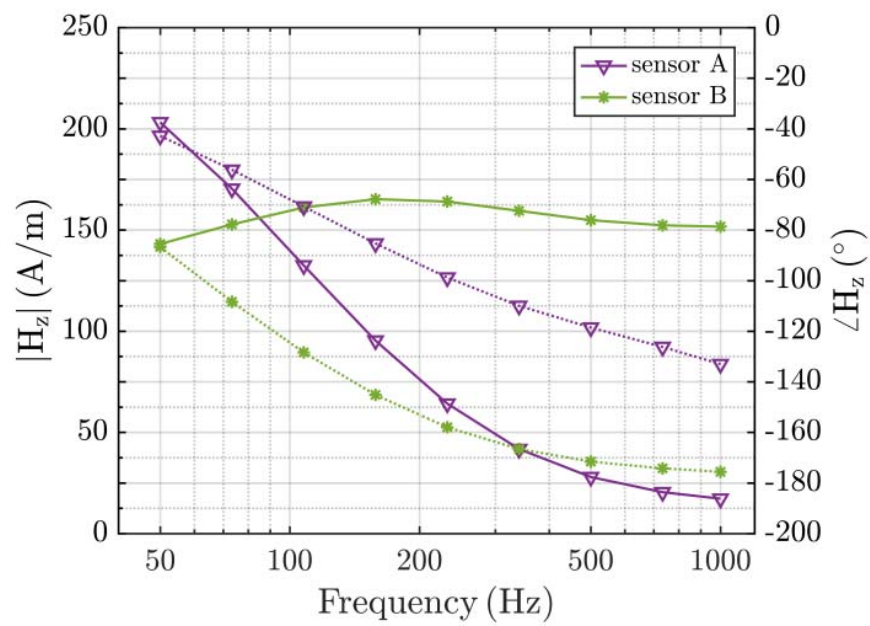

Fig. 7. Simulated frequency dependence of individual sensors.

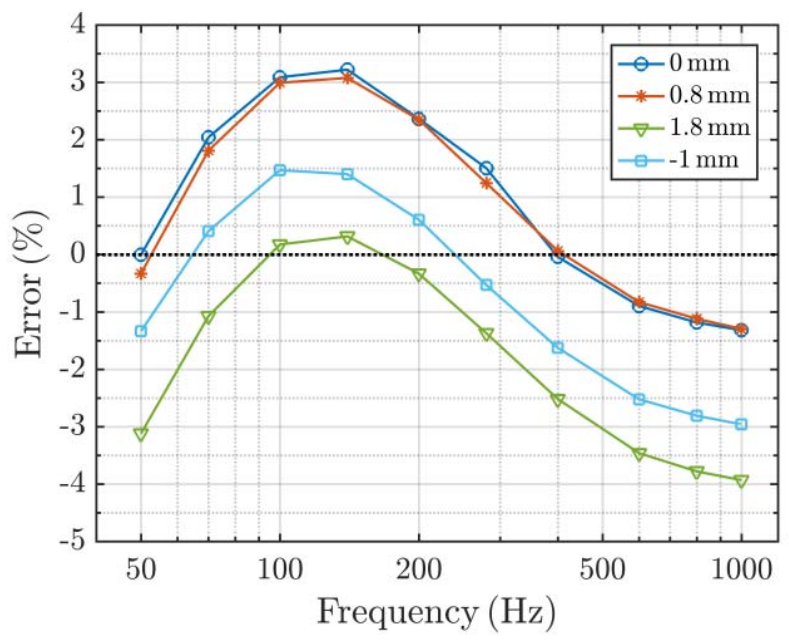

Fig. 8. Measured frequency characteristics for several values of the vertical position of the sensors in the hole. The graph shows deviation from sensitivity at $50 \mathrm{~Hz}$.

frequency error up to $1 \mathrm{kHz}$ is below $3 \%$. The phase error was only slightly reduced to $8^{\circ}$ at $1 \mathrm{kHz}$, but up to $600 \mathrm{~Hz}$, the phase characteristics is linear.

Fig. 7 shows the principle of the compensation mechanism: the frequency dependence of the individual sensors is high. The differences are caused by different effect of eddy currents in each point. In this way, the frequency dependence of the differential signal is dramatically decreased. If we vectorially subtract voltages for A and B sensors, we obtain theoretical characteristics shown in Fig. 6.

The compensation technique based on the subtraction of two similar variables raises the question of the stability of this compensation in real conditions. We therefore studied the stability of the sensor with temperature and geometrical tolerances.

\section{Sensor Stability And Resistance TO EXTERNAL CURRENTS}

Fig. 8 shows how the frequency characteristics change with vertical sensor misalignment. It is clear that the changes in the shape of the frequency characteristics are negligible, but the 


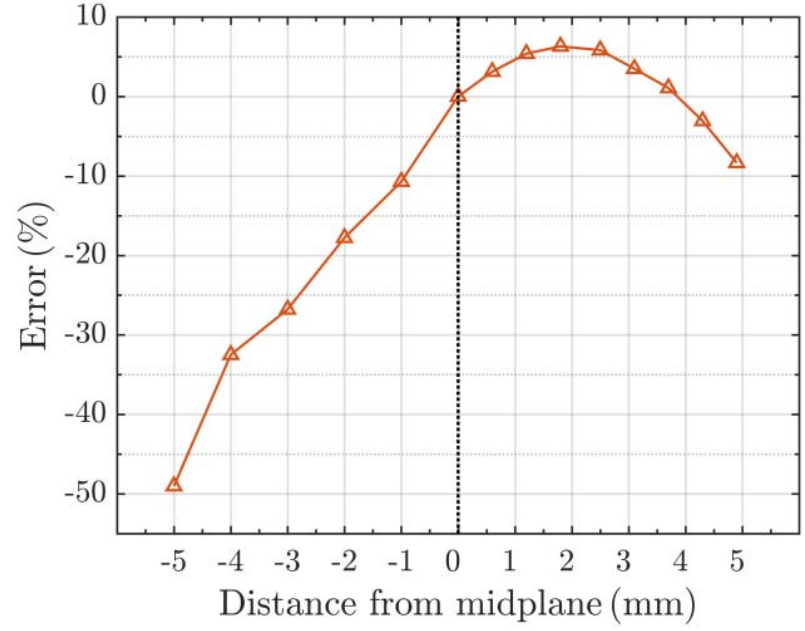

Fig. 9. Dependence of the sensitivity on the vertical position of the sensors in the hole. The graph shows deviation from sensitivity at the central point.

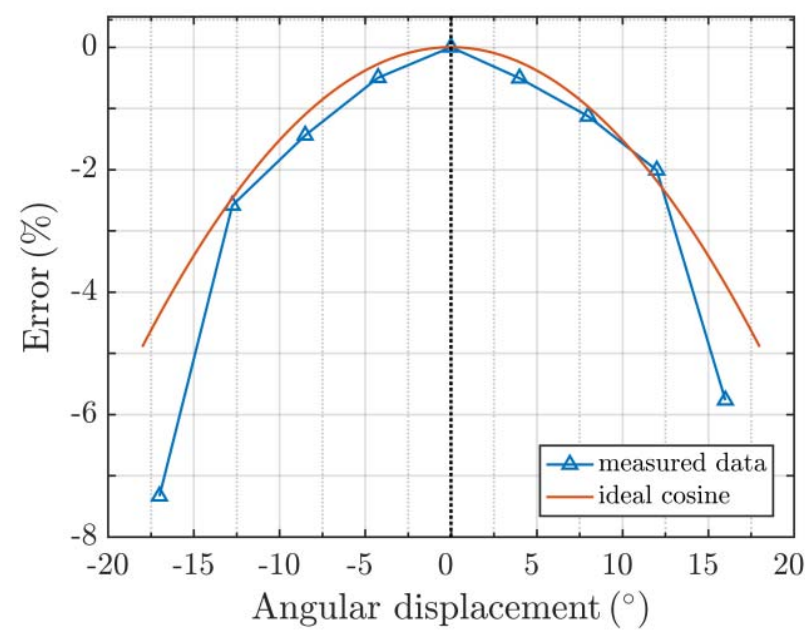

Fig. 10. Sensitivity error caused by rotational displacement of the sensor. The theoretical error caused by cosine dependence is shown for comparison.

sensitivity depends on the vertical position of the sensor, as shown in Fig. 9. The maximum sensitivity point is not in the central plane, as for a simple busbar sensor, but $2 \mathrm{~mm}$ above. In this optimum location, the sensitivity to position error is also minimized.

Fig. 10 shows the sensitivity to rotational displacement of the differential sensor. It is clear that the characteristics differ significantly from the cosine shape. By linear approximation, we may estimate that $0.2^{\circ}$ angular displacement caused by temperature cycling would cause only $0.025 \%$ error, which is negligible in comparison with the $0.1 \%$ linearity error of the device.

We also examined the effect of self-heating by FEM simulations. At a maximum current of $1000 \mathrm{~A}$, the temperature of the current bar is $50{ }^{\circ} \mathrm{C}$. The sensitivity change caused by temperature effects is $1 \%$. In comparison with this, the sensitivity change with the temperature of the fluxgate sensor itself is only $7 \mathrm{ppm} /{ }^{\circ} \mathrm{C}$.

The dc offset stability depends mainly on the parameters of the fluxgate sensor. The specified maximum drift of $5 \mathrm{nT} /{ }^{\circ} \mathrm{C}$ would correspond to $2.5 \mathrm{~mA} /{ }^{\circ} \mathrm{C}$. The dc current resolution is limited to $10 \mathrm{~mA}$, as the sensor noise power spectrum density is $2 \mathrm{~mA} / \sqrt{ } \mathrm{Hz}$ at $1 \mathrm{~Hz}$.

With a sensitivity of $500 \mathrm{~A} / \mathrm{mT}$, the calculated effect of an external $1000 \mathrm{~A}$ current at a distance of $15 \mathrm{~cm}$ is $3.4 \mu \mathrm{T}$, so the error is only $0.17 \%$. We measured the influence of the external current in the real busbar and found a very similar error of $0.15 \%$.

\section{CONCLUSion}

The busbar fluxgate current sensor presented here has very small ferromagnetic cores inside the two integrated feedback-compensated fluxgate sensors. This keeps the power consumption of our device below $100 \mathrm{~mW}$, even for the maximum measured current of $1000 \mathrm{~A}$. This is much lower than the power consumption of around $15 \mathrm{~W}$, even when using very efficient electronics, for another class of feedbackcompensated fluxgate current sensors with large cores around the measured current conductor [13].

In addition to the advantages of the yokeless busbar current sensor, which are its small size, lightweight, and low power consumption, we also should mention its disadvantages. The sensor needs to be inserted into the measured circuit, which is not practical for ambulatory measurements. Compared with that, the yoke can be made openable as clamps. A yoke is also believed to better suppress the influence of external fields, but we have shown that the small distance of our differential sensor pair performs similarly, as the error for an external current at a distance of $15 \mathrm{~cm}$ is only $0.15 \%$.

The new shape of the busbar sensor improved the frequency characteristics: the achieved error was $\pm 3 \%$ in amplitude and $8^{\circ}$ in phase at $1 \mathrm{kHz}$. The sensor linearity is $0.1 \%$, in comparison with $1 \%$ in [9]. With $1000 \mathrm{~A}$ range, the sensor has $10 \mathrm{~mA}$ resolution and $2.5 \mathrm{~mA} /{ }^{\circ} \mathrm{C}$ maximum dc drift. The temperature stability is, therefore, four times better than when using an AMR sensor and 1000 times better than when using Hall sensors. The external current in a $9 \mathrm{~cm}$ distant busbar is suppressed by a factor of 66 .

\section{ACKNOWLEDGMENT}

The authors would like to thank A. Chirtsov, who performed some of the measurements. A. Chirtsov and V. Grim received student support from Texas Instruments, which also supplied the sensor boards.

\section{REFERENCES}

[1] P. Ripka, "Electric current sensors: A review," Meas. Sci. Technol., vol. 21, no. 11, pp. 1-23, Sep. 2010.

[2] G. Velasco-Quesada, M. Roman-Lumbreras, A. Conesa-Roca, and F. Jerez, "Design of a low-consumption fluxgate transducer for highcurrent measurement applications," IEEE Sensors J., vol. 11, no. 2, pp. 280-287, Feb. 2011.

[3] X. Yang, Y. Li, W. Zheng, W. Guo, Y. Wang, and R. Yan, "Design and realization of a novel compact fluxgate current sensor," IEEE Trans. Magn., vol. 51, no. 3, pp. 1-4, Mar. 2015.

[4] BBM. Current Sensors Manufactured by Senis, Catalogue, accessed Nov. 4, 2016. [Online]. Available: http://www.senis.ch/

[5] M. Blagojević, U. Jovanović, I. Jovanović, D. Mančić, and R. S. Popović, "Realization and optimization of bus bar current transducers based on Hall effect sensors,” Meas. Sci. Technol., vol. 27, no. 6, p. 065102, 2016. 
[6] K.-L. Chen and N. Chen, "A new method for power current measurement using a coreless Hall effect current transformer," IEEE Trans. Instrum. Meas., vol. 60, no. 1, pp. 158-169, Jan. 2011.

[7] F. Koga, T. Tadatsu, J. Inoue, and I. Sasada, "A new type of current sensor based on inverse magnetostriction for large current detection," IEEE Trans. Magn., vol. 45, no. 10, pp. 4506-4509, Oct. 2009.

[8] M. F. Snoeij, V. Schaffer, S. Udayashankar, and M. V. Ivanov, "Integrated fluxgate magnetometer for use in isolated current sensing," IEEE J. Solid-State Circuits, vol. 51, no. 7, pp. 1684-1694, Jul. 2016.

[9] Z. Zhenhong, O. Syuji, A. Osamu, and K. Hideto, "Development of the highly precise magnetic current sensor module of $+/-300$ A utilizing AMR element with bias-magnet," IEEE Trans. Magn., vol. 51, no. 1, pp. 1-5, Jan. 2015.
[10] P. Ripka, M. Přibil, V. Petrucha, V. Grim, and K. Draxler, "A Fluxgate Current Sensor With an Amphitheater Busbar," IEEE Trans. Magn., vol. 52, no. 7, Jul. 2016, Art. no. 4002004.

[11] DRV425 Fluxgate Magnetic-Field Sensor, Texas Instruments Datasheet, accessed Nov. 4, 2016. [Online]. Available: http://www.ti.com/product/DRV425/datasheet

[12] P. Ripka, K. Draxler, and R. Styblikova, "AC/DC current transformer with single winding," IEEE Trans. Magn., vol. 50, no. 4, Apr. 2014 Art. no. 8400504.

[13] G. Velasco-Quesada, M. Román-Lumbreras, R. Pérez-Delgado, and A. Conesa-Roca, "Class $\mathrm{H}$ power amplifier for power saving in fluxgate current transducers," IEEE Sensors J., vol. 16, no. 8, pp. 2322-2330, Apr. 2016. 